\title{
47. Studies on Homocarnosine as a Inhibitory Substance in Cerebral Cortex
}

\author{
Dennosuke JinnaI, Akitane Mori and Masanori KaWANISHI \\ Dept. of Neurological Surgery, Osaka University Medical School \\ Riyosuke Sugiu, Sadahiko MasuKawa and Shintaro MiYake \\ Ist Dept. of Surgery, Okayama University Medical School
}

Homocarnosine is a dipeptide which was isolated from beef brain in amounts of about 0.5 to $1 \mathrm{mg}$ per $100 \mathrm{~g}$ of tissue by Pisano et al. (1960), and chemical structure of it was identitied as $\gamma$-aminobutyryl-L-histidine by them. Recently it was found that homocarnosine is a inhibitory substance of cerebral cortex by us, i.e. the typical convulsive seizure of ep-mouse was completely inhibited by means of intra-thecally administration of homocarnosine and simultaneously spike waves observed in EEG findings of ep-mouse were vanished. The other hand, convulsion due to sodium citrate was inhibited too by it. Generally, homocarnosine may be a inhibitory substance in limbic system, as suggested by Dr. Hayashi.

Most recently synthesis of ${ }^{14} \mathrm{C}$-labelled homocarnosine from ${ }^{14} \mathrm{C}-\gamma$-aminobutyric acid was done successively by us, and we are planning to research its metabolism.

\author{
48. Treatment of Epileptic Patients by the Candidate \\ Substances of the Inhibitory Transmitter in the \\ General Nervous System \\ Mutsutoshi KosaKa \\ Dept. of Neurology, Okayama University Medical School
}

\author{
Akira Nishimoto \\ Dept. of Surgery, Okayama University Medical School
}

Hiroo TAKashita

Dept. of Physiol., Nihon Univ. School of Dent.

Hisao Namba, Hiroyuki Ohnishi, Fukujiroh Miki, Shizuko Namba, and Daizo Nagano

Okayama Institute of Epilepsy

Hayashi has discussed that $\gamma$-amino- $\beta$-hydroxybutyric acid (GABOB) is a 
candidate substance to inhibitory chemical transmitter of the central nervous system of higher animals, if so, this substance could be used therapeutically in the treatment of epilepsy.

1) We have had a three years observation on 65 patients. One third of them stopped seizures completely within the period of medication, but to our unsatisfaction other two thirds did not stop, however, improved.

2) Next we injected a rather concentrated GABOB into the cerebrospinal fluid through the lumber punction and pumped it several times, so as to mix it with c.s.f. of the higher parts of nervous system. The result was that two thirds of the patients stopped seizures for some weeks, but the other one third remained unchanged.

3) Hayashi has found that there are two pathways of producing seizure, a system from the pallial cortex, the other from the limbic system, and GABOB was chemical inhibitor of the former. Hayashi pointed that inhibitory transmitter of limbic system should be Carnosine or Homocarnosine. We introduced GABOB with Hocoarnosine into c.s.f. of patients through lumber punction, and gave several times pumpings. The results were remarkable and complete.

\title{
49. Forel-H Tomy for Epileptic Convulsion (Part 2)
}

\author{
Dennosuke JinNaI \\ *2nd Surgical Clinic, Osaka University Medical School \\ Akira Nishimoto, Shuzo Okumura, Saburo KaWata, Toru Higashi, \\ Jiro Mukawa, Haruo Suzuki and Toshio Hiroe \\ *Dept. of Neurosurgery, Okayama University Medical School
}

Further basic study of Forel-H tomy was performed with cat. The Forel-H lesion rised metrazol threshold remarkablly for epileptic convulsion, but same size lesion in the neighboring structure to Forel-H field failed to prove the rising threshold.

By clinical experience, 45 advanced epileptics underwent Forel-H oil-wax lesion, 30 cases revealed marked or considerable decreasing of frequency and duration of convulsion on the follow up data longer than 6 months.

Neither specific electrical ativity by deep electrode (deep EEG.) in Forel-H field, nor modification of cerebral cortical activity by Forel-H stimulation were observed in the clinical cases. 